\title{
LOCALISATION OF THE LEVEL OF SYMPTOMATIC CERVICAL DISC DEGENERATION
}

\author{
SHINICHI KIKUCHI, IAN MACNAB, PAUL MOREAU
}

From the Wellesley Hospital, Toronto, Canada

\begin{abstract}
There are many difficulties associated with the localisation of the symptomatic segment in patients presenting with cervicobrachial pain with no evidence of impaired conduction in the nerve root. Ancillary radiological investigations such as myelography, epidural phlebography, and epidural myelograms are of unreliable diagnostic value.

However, discography can be of value if the technique described here is used. Infiltration of the cervical nerve root with local anaesthetic has also proved useful in the localisation of the symptomatic segment. The techniques used in cervical discography and infiltration of the nerve root are described and their reliability is assessed.
\end{abstract}

There is increasing doubt concerning the efficacy of anterior cervical fusion in the treatment of cervical disc degeneration in patients with no evidence of impaired root conduction. This is due to the difficulty in localising the symptomatic segment or segments. The radiological evidence of marked degeneration in a segment does not necessarily indicate that this level is the source of symptoms (Figs 1 and 2). The majority of patients with severe neck pain do not show instability of the segment when radiographs of the cervical spine are taken in full flexion and full extension of the neck. The demonstration of excessive or irregular mobility does not necessarily mean that the symptomatic segment has been located.

In patients with no evidence of root compression, myelography is not a reliable indication of the pathological level. Over the past years it has been our policy to screen and examine the whole spine when performing lumbar myelography: in the cervical spine myelographic defects are commonly found in patients without symptoms and may be present at several levels (Fig. 3). Epidurography using a water-soluble contrast material is used to define the nerve roots (Figs 4 and 5) and clearly demonstrates lack of filling of obstructed root sleeves (Fig. 6). However, clear radiological changes of this type are invariably associated with clinical evidence of impaired conduction in the root. This technique therefore has little value in the localisation of the symptomatic segment in patients who do not show conduction deficits.

Cervical epidural phlebography clearly demonstrates the presence of neurocentral osteophytes (Fig. 7) which are also defined by CAT scanning. The presence of such changes gives no indication of their clinical significance.

Discography and infiltration of the cervical nerve root with local anaesthetic have been of value in determining the symptomatic segment in this study.

\section{CLINICAL MATERIAL}

The 138 patients studied had suffered from intermittent cervicobrachial pain for more than two years before operation. In 41 patients the main symptom was pain in the neck with occasional radiation of pain to the shoulder, occiput or chest. In the remaining 97 patients the pain in the neck was associated with pain radiating down the arm. In none of the 138 patients was there any evidence of impaired conduction in the root. Fusion of a single segment was carried out on every patient. This operation involved excision of the disc, removal of the adjacent end plates of the vertebral bodies and the insertion of a block of bone to give maximal distraction of the vertebral bodies.

\section{METHODS}

Cervical discography. Cervical discography was introduced by Cloward in 1958 and this technique has been subjected to criticism. Degenerative patterns revealed by discography are common in patients over the age of $\mathbf{4 0}$ years and often involve several segments. Demonstration of an anatomical abnormality in the disc therefore has no clinical significance. Since the radiographic appearance is not of diagnostic significance, this technique is more accurately termed discometric analysis, but to avoid confusion the term discography will be used. However, reproduction of the clinically experienced pain when the involved segment is manipulated during discography is of diagnostic value. A second disadvantage of this technique is that a normal disc may be painful when a large volume of dye is injected under pressure (Holt 1964)

S. Kikuchi, MD. Clinical Research Fellow
I. Macnab. MD. FRCS. Chief of Orthopaedic Surgery $\}$ The Wellesley Hospital, 160 Wellesley Street East, Toronto, Ontario, Canada P. Moreau. MD. FRCS. Clinical Research Fellow $\mathrm{M} 4 \mathrm{Y} 1 \mathrm{~J} 3$

Requests for reprints should be sent to Dr I. Macnab, Room 307. The E. K. Jones Building, 160 Wellesley Street East. Toronto, Ontario, Canada M4Y $1 \mathrm{~J} 3$.

(1) 1981 British Editorial Society of Bone and Joint Surgery 0301-620X/81/2052-0272 $\$ 2.00$ 


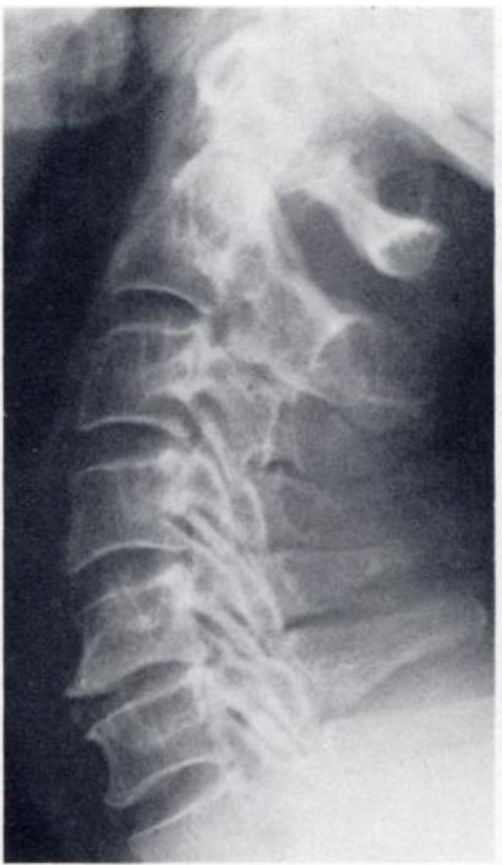

Fig. 1

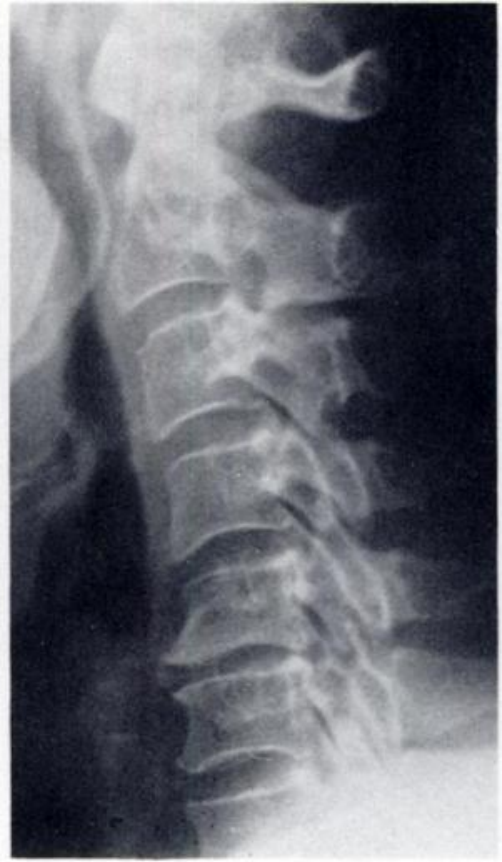

Fig. 2

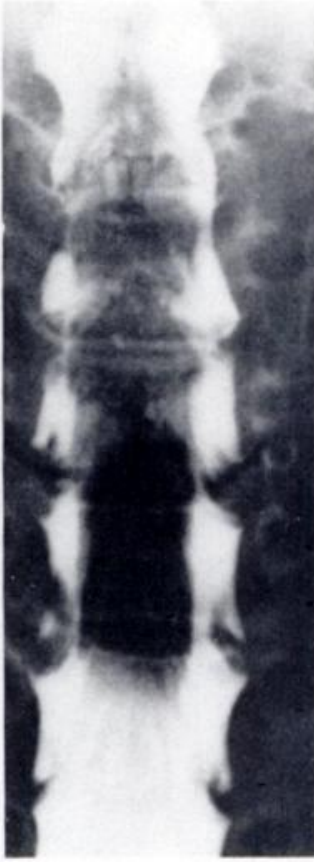

Fig. 3

Figures 1 and 2-Radiographs showing degeneration in the $\mathrm{C} 5$ and $\mathrm{C} 6$ segment. The disc between $\mathrm{C} 5$ and $\mathrm{C} 6$ has lost height; the adjacent vertebral bodies show reactive spondylophytes and on flexion and extension this segment does not move. Discography indicated the C4 and C5 segment to be the cause of pain which was subsequently relieved by fusion of this segment. Figure 3-Radiograph of a 54-year-old patient who had never suffered from any cervicobrachial pain. Routine screening of the cervical spine after lumbar myelography revealed bilateral flow defects at several segments.

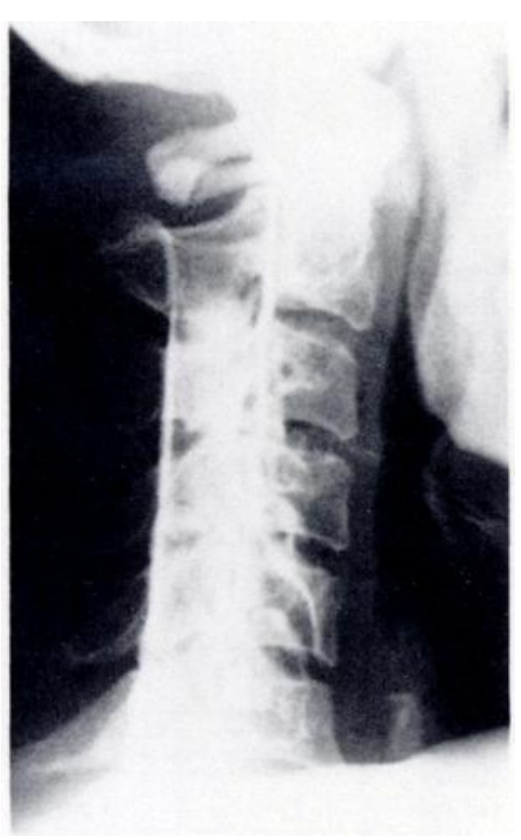

Fig. 4

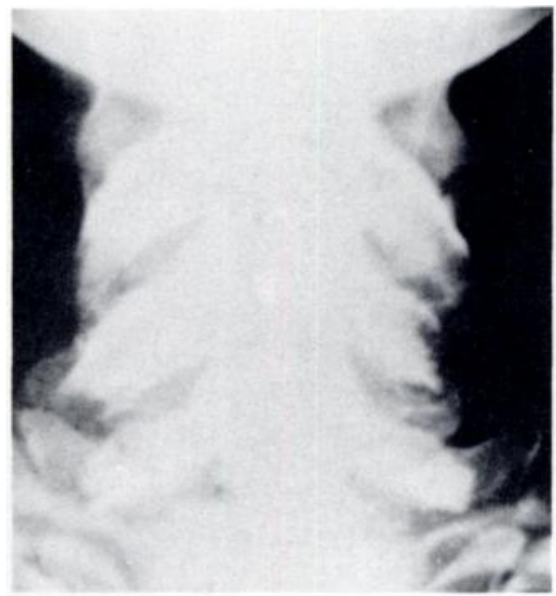

Fig. 5

Figure 4-Epidural myelograph to show the position of the contrast material (metrizamide) on the lateral view. Figure 5Epidural myelograph to show the root sleeve filling. Figure 6-Epidural myelograph showing the inability to fill the $\mathrm{C} 6$ root sleeve with contrast medium. This patient had pain in both arms and clinical evidence of impaired conduction along the $\mathrm{C} 6$ root.

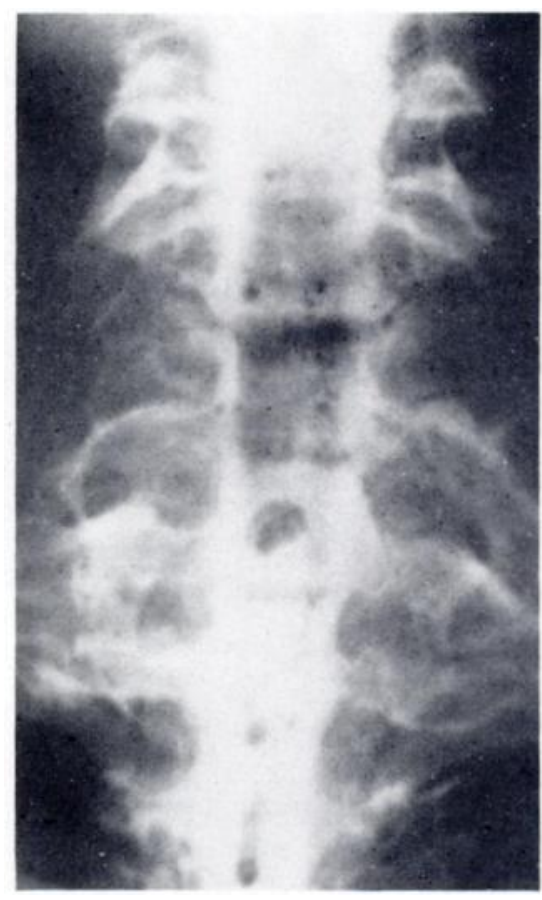

Fig. 6
To make use of discography a meticulous procedure must be followed. First the anterior longitudinal ligament must be infiltrated carefully with a local anaesthetic. This ligament may be an irritable focus in the presence of degenerative disc disease and the injection of an innocuous disc by causing pressure on the anterior longitudinal ligament may give rise to pain. After the ligament has been infiltrated with local anaesthetic the needles are inserted into at least four adjacent discs. It is essential that the tip of the needle is placed in the middle of the nucleus and its position confirmed by the injection of a small quantity (less than 0.25 millilitre) of a water-soluble radioopaque dye. If the needles are correctly placed then the discs are distended by injecting one per cent lidocaine. By using a local 
anaesthetic as the distending agent any pain produced is of short duration. The injection is stopped as soon as any discomfort is experienced by the patient and no more fluid is injected until the discomfort subsides.

This technique enables re-examination of the segments that were painful by means of a further injection of lidocaine. If the pain produced is minor and of short duration then the disc can be re-injected with saline which produces more discomfort. This makes it easier for the patient to recognise the similarity of the pain caused by injection and the pain normally experienced. Discography therefore

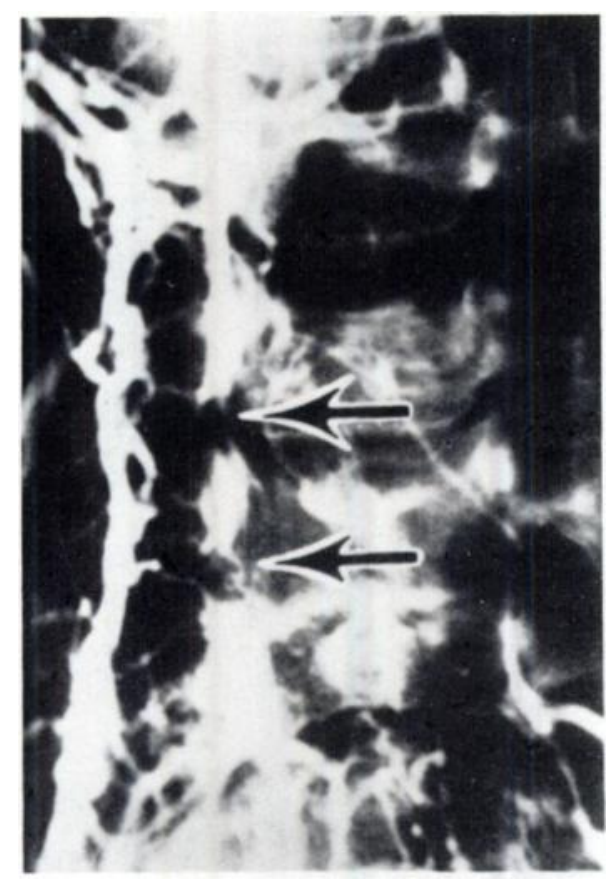

Fig. 7

Cervical phlebograph showing marked defects in the flow of the anterior internal vertebral veins in the C4. C5. C6 area. These defects are due to neurocentral osteophytes. The test is too sensitive to be of diagnostic value.

gives a clear indication of the segment or segments causing the painful response.

It is very difficult to determine the accuracy of any diagnostic technique. Simmons. Bhalla and Butt (1969) regarded freedom from symptoms after solid fusion an indication that the correct segment had been fused. In their series 30 of the 31 patients obtained relief of pain when fusion was performed at the level where pain was experienced during discography. However, the end results are not necessarily related to the solid fusion achieved as other factors such as the passage of time, changes in lifestyle and change of job may contribute to the successful results.

It is commonly found that after an anterior cervical fusion many patients will have relief of symptoms immediately after the operation whilst still in the recovery room. If there is no relief at this time then the result is usually unsatisfactory (Table I). It was therefore decided to use the assessment of the immediate relief of pain by the patient after operation as an indication of the diagnostic accuracy of discography. To reduce the number of variables the study was confined to emotionally stable patients in whom the clinical symptoms were reproduced by the injection of a single segment.

Infiltration of the root sleeve. Infiltration with local anaesthetic has proved to be of diagnostic value in the assessment of patients with continuing sciatic pain after previous spinal operation (Macnab 1971). This pain is temporarily abolished when the nerve root involved is infiltrated with local anaesthetic. It is possible to use a similar technique in the diagnosis of patients suffering from cervicobrachial pain. Abolition of the pain in the arm after injection of local anaesthetic into the sleeve of the root indicates the segment involved.

In the cervical spine, unlike the lumbar spine, the nerve roots emerge through the intervertebral foramina above the level of the segment (Fig. 8). The roots emerge from their foramen and lie anterolaterally in the costotransverse canal where they emerge posterior to the vertebral artery.

Recognition of the epiradicular sheath is important in the infiltration of the nerve root. In our dissections of the epidural space of the cervical spine the epidural membrane was found to surround the dura (Frykholm 1951; Cailliet 1964; Hayashi et al. 1977). On the dorsal side the epidural membrane is attached to the ligamentum flavum and the lamina. Anteriorly the superficial layer of the posterior longitudinal ligament blends with the epidural membrane. It is thickened around the nerve root and forms a fibrous covering called the epiradicular sheath (Figs 9 and 10). This sheath has a loose attachment to the nerve root but is firmly attached to the periosteum of the transverse process and the scalene group of muscles. The sheath

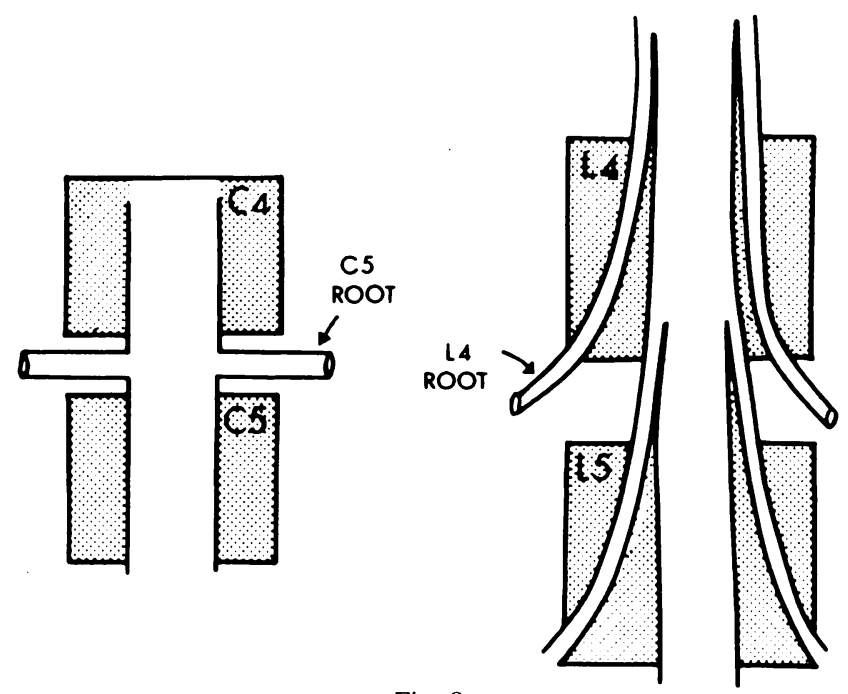

Fig. 8

Comparison of the points at which the nerve roots emerge from the cervical spine and the lumbar spine.

continues as a loose connective tissue around the peripheral nerves forming the epineural sheath at the cervical plexus.

When infiltrations of the cervical nerve root were performed on dog cadavers the contrast material spread peripherally under the epiradicular and epineural sheath. Some of the injected contrast material spread proximally and lay between the epidural membrane and the dura. Similarly when infiltration was performed on human

Table I. The results for 54 patients in whom discography was not performed before the anterior cervical fusions

\begin{tabular}{|l|c|c|c|}
\hline & \multirow{2}{*}{$\begin{array}{c}\text { Results immediately } \\
\text { after operation }\end{array}$} & $\begin{array}{c}\text { Number of } \\
\text { patients }\end{array}$ & \multicolumn{2}{|c|}{ Result one year after fusion } \\
\cline { 3 - 4 } & 38 & 28 & No pain \\
\hline No relief of pain & 16 & 5 & 10 \\
Relief of pain & 38 \\
\hline
\end{tabular}

cadavers the contrast material spread proximally under the epidural membrane and also distally under the epiradicular sheath. The epiradicular sheath causes the contrast material to flow in a tubular fashion.

Technique for infitration of the root sleeve. In clinical practice the needle is inserted against a nerve root after it has emerged through the 


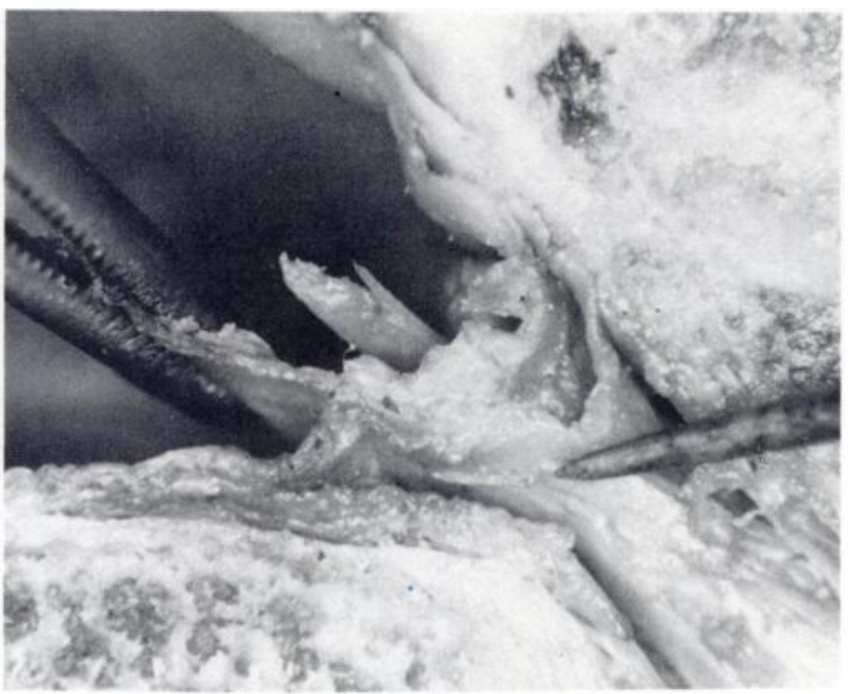

Fig. 9

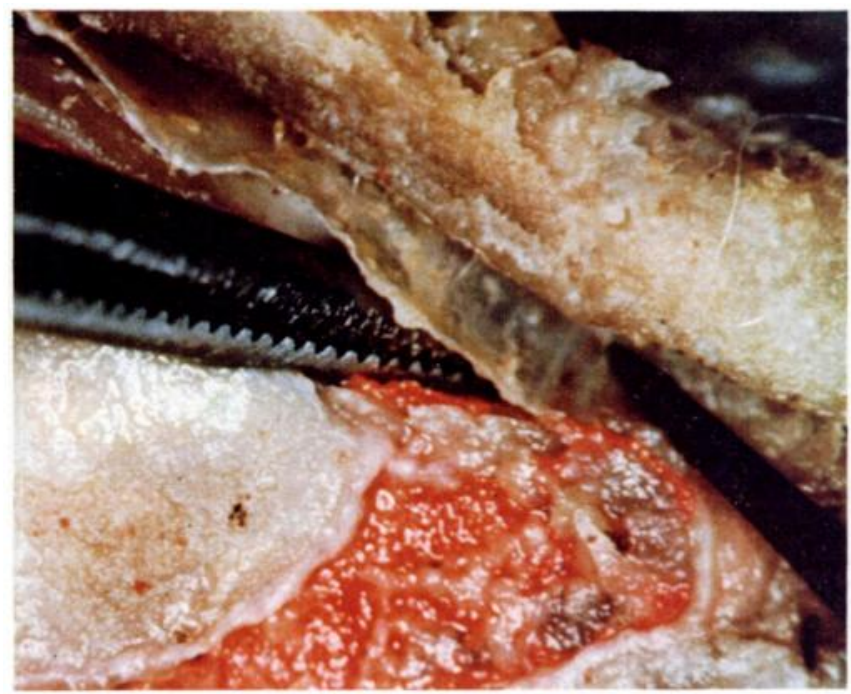

Fig. 10

Figure 9-The dura is held by forceps and the pointer indicates the condensation of the epidural membrane forming the epiradicular sheath at the point of emergence of the root. Figure 10-A dissection showing the epidural membrane and its continuity with the periradicular sheath. The epidural membrane is seen above the forceps and the periradicular sheath is over the tip of the forceps.

foramen. A small quantity of water-soluble radio-opaque dye is injected to ensure that the needle has been placed correctly. If the needle has been correctly positioned the dye will spread in a tubular fashion and will be clearly outlined on the radiograph (Fig. 11). Incorrect placement of the needle causes the dye to form a diffuse blob on the radiograph rather than the typical tubular shape. Three approaches may be used when inserting the needle: anterior, lateral and posterior (Fig. 12).

Anterior approach. This approach is used in discography. The carotid sheath is pulled laterally by the fingers which are placed into the sulcus between the sheath and midline structures. The needle is inserted as far as the tip of the transverse process immediately lateral to the vertebral artery (Fig. 13).

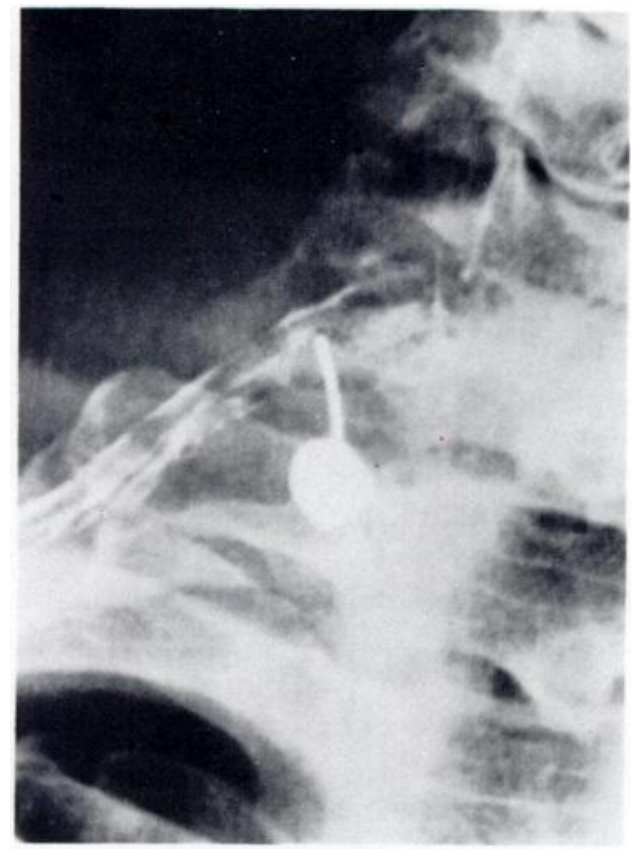

Fig. 11

Radiograph to show the cervical nerve root outlined by the injection of metrizamide into the periradicular sheath.
Lateral approach. The patient is placed in the supine position with the chin pointing upwards and the transverse process is identified using the fingers. The needle is then entered 0.5 centimetre anterior to the line joining the tip of the mastoid process to the tubercle of Chassaignac and directed to hit the transverse process at one level above the suspected root. The needle is then withdrawn and directed downwards and medially. This technique avoids the danger of the needle being inadvertently inserted into the intervertebral foramen.

Posterior approach. The patient is placed on one side with the affected extremity uppermost. To inject the $\mathrm{C} 3, \mathrm{C} 4, \mathrm{C} 5$, and $\mathrm{C} 6$ nerve roots the needle is inserted at an angle of 45 degrees five centimetres from the midline and is advanced until it touches the transverse process. To inject the $\mathrm{C} 7$ and $\mathrm{C} 8$ nerve roots the needle is inserted seven centimetres from the midline at an angle of 60 degrees. The injection of these $\mathrm{C} 7$ and $\mathrm{C} 8$ roots by the posterior approach is very difficult because of the thickness of the tissues between the site of the puncture and the transverse process.

There are three possible complications associated with infiltration of a cervical nerve root which were not encountered in this series. It is possible to puncture a major blood vessel; to penetrate the dura and cause injury of the spine; or to penetrate the cupola and give rise to a pneumothorax. To avoid these complications it is important that the superficial guide points are identified. Gentle suction may be applied to the needle before injection of the contrast material to ensure that neither blood nor cerebrospinal fluid is withdrawn.

In this study the anterior approach was used with every patient. When the needle strikes the nerve root involved the pain is reproduced. A small quantity of radio-opaque dye is then injected to confirm that the needle has been correctly placed. The loss of symptoms after injection of one millilitre of one per cent lidocaine confirms the level of the lesion.

\section{RESULTS}

In the recovery room after anterior cervical fusion 114 patients ( 82.6 per cent) stated that they were no longer conscious of their symptoms. On re-examination of this group one year later 110 patients were either totally free of symptoms or were conscious of discomfort which did not interfere with their work or their social life. Two of the four patients with significant residual symptoms had developed a pseudarthrosis. 


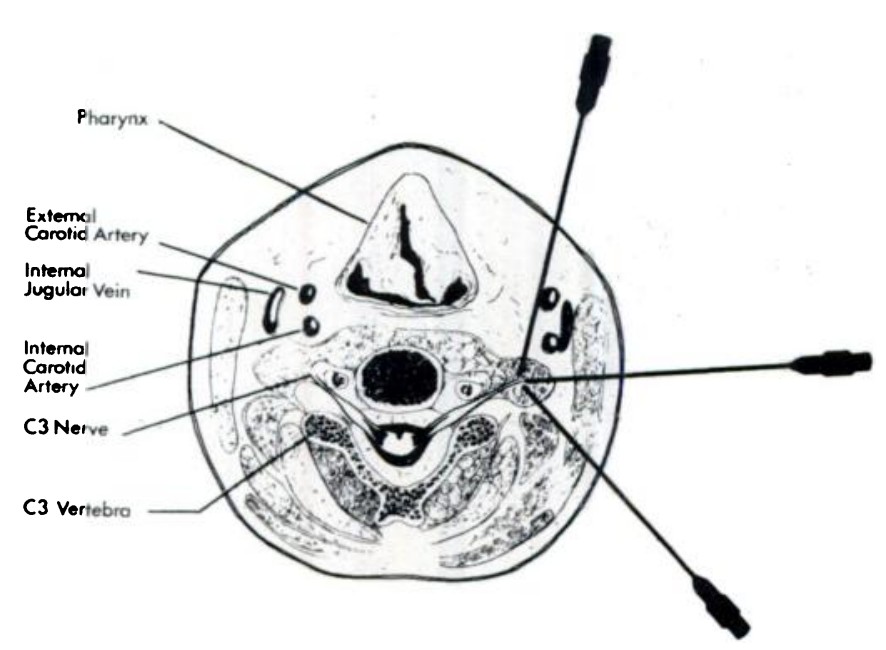

Fig. 12

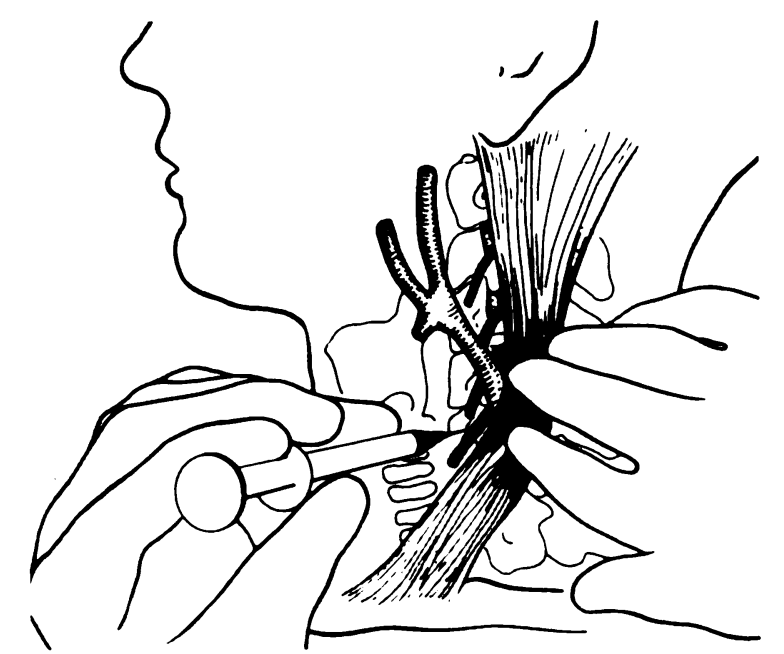

Fig. 13

Figure 12-Diagram to show the anterior, lateral and posterior approaches used in the infiltration of the cervical root. Figure 13-Infiltration of the cervical nerve root using the anterior approach. The tips of the fingers are placed in the sulcus between the anterior border of the sternum mastoid and the midline structures. The needle is then inserted along the line of the fingers down to the transverse process striking the roots emerging lateral to the vertebral body.

The immediate relief of pain after operation and the good results one year later indicate the accuracy of cervical discography in determining the symptomatic segment.

Seventy-five patients in whom infiltration of the root sleeve was employed were reviewed. The first 10 patients analysed demonstrated clinical evidence of impaired conduction of a cervical nerve root and also a clearly defined defect on myelography. These 10 patients were of value in assessing the accuracy of the technique. The C6 root was involved in six patients of this group and the $\mathrm{C} 7$ root involved in the other four. Infiltration of the involved root temporarily abolished the pain in all 10 patients, and all acknowledged relief of pain after anterior cervical discectomy and fusion.

The remaining 65 patients with cervicobrachial pain had no clinical evidence of impaired conduction in the roots. In 29 patients myelography was performed. Six of these 29 patients showed a defect at a single level and in four of these the pain in the arm could be abolished by infiltration of the nerve root at the segment showing the myelographic defect. In the remaining two the pain in the arm was relieved by injection of the root at the segment below the myelographic defect. In 12 patients the myelographs were normal and in the other 11 significant defects were demonstrated at two or more levels. In these latter patients an attempt was made to determine the symptomatic level by discography and infiltration of the root. In every patient when discography reproduced the symptoms infiltration of the nerve root abolished the pain in the arm.

As with discography the immediate result after operation was used to assess the accuracy of the root sleeve infiltration as a diagnostic test. Immediate relief of pain in the arm after operation, before an analgesic was given. indicated that the correct segment had been operated upon.

Sixty-five of the 75 patients, in whom the symptomatic level was assessed by infiltration of the root, experienced relief of pain in the arm whilst still in the recovery room after anterior cervical fusion. Although eight of the 10 remaining patients subsequently improved, factors other than accurate localisation of the lesions had to be taken into consideration when assessing the results.

\section{DISCUSSION}

Spinal fusion in the lumbar region is an accepted treatment for patients who continue to be disabled by a discogenic pain in the lower back and who fail to respond to conservative therapy. These patients have no evidence of irritation of a root. In the cervical spine it has been stated that anterior cervical fusion is only indicated with evidence of impaired root conduction in one or two segments. This view developed as it was virtually impossible to demonstrate the symptomatic segment by routine radiography, flexion and extension studies, myelography, phlebography or epidurography.

With the introduction of discography it was hoped that a better technique for identifying the symptomatic segment would be available. It soon became apparent that the radiographic demonstration of disc degeneration was not a reliable diagnostic sign. It was also noted that the anterior and posterior ligaments are very sensitive and the injection of a tissue irritant, such as a water soluble contrast material, could produce pain when injected into a clinically asymptomatic disc. If the tip of the needle is advanced so it is touching the cartilaginous end-plate then the injection of contrast material will cause pain. It is essential that the needle is placed accurately in the centre of the nucleus. 
Since pain could be produced by the hydraulic manipulation of a single segment it became apparent that diagnostic discography could be performed with a non-irritating solution such as normal saline or local anaesthetic. The technique was made more sensitive by the preliminary infiltration of the anterior longitudinal ligament with local anaesthetic.

Due to the presence of a periradicular sheath and an epidural membrane it was possible to infiltrate the root with local anaesthetic. Clinically it could be shown that patients suffering from cervicobrachial pain could be relieved of the radiating pain in the arm by anaesthetis- ing the nerve root at the symptomatic segment, even if there was no clinical evidence of impaired conduction in the roots.

In clinical practice, the pathological state of the cervical discs is first determined by discography. If the patient suffers from pain radiating down the arm in addition to pain in the neck, further confirmation of the segment involved can be obtained by infiltration of the nerve root. Using these diagnostic techniques cervical fusion becomes a rational and logical treatment for segmental instability in the absence of evidence for compression of the root.

The authors wish to express their thanks to the Workmen's Compensation Board of Ontario for their financial support for this study. We would also like to express our gratitude to Professor T. Ito. Chief of the Department of Anatomy, Fukushima Medical College for his advice and his assistance in carrying out the necropsy studies.

\section{REFERENCES}

Cailliet R. Neck and arm pain. Philadelphia: FA Davis, 1964.

Cloward RB. Cervical diskography, technique, indications and use in diagnosis of ruptured cervical disks. AJR 1958;79:563-74.

Frykholm R. Cervical nerve root compression resulting from disc degeneration and root sleeve fibrosis. Acta Chir Scand 1951;Suppl 160.

Hayashi K, Yabuki T, Kurokawa T, Seki H, Hogaki M, Minoura S. The anterior and posterior longitudinal ligaments of the lower cervical spine.J Anat 1977;124:633-6.

Holt EP Jr. Fallacy of cervical discography. JAMA 1964;188:799-801.

Macnab I. Negative disc exploration. J Bone Joint Surg $[A m]$ 1971;53-A;891-903.

Simmons EH, Bhalla SK, Butt WP. Anterior cervical discectomy and fusion.J Bone Joint Surg [Br] 1969;51-B:225-37. 